\title{
Calidad de Vida Profesional y Burnout en Técnicos de Drogodependencias: Análisis Comparativo con otros Grupos Profesionales
}

\author{
Professional Life Quality and Burnout Among \\ Substance Abuse Technicians: A Comparative \\ Analysis with other Professional Groups
}

\author{
Ignacio García Barquero \\ Médico. Centro de Atención a las Drogodependencias \\ de San Blas, Madrid. España. \\ Macarena Gálvez Herrer \\ Psicóloga. Técnico Superior en P.R.L. Unidad de Valoración y Orientación \\ al Profesional Sanitario Enfermo (UVOPSE-PAIPSE) \\ Consejería de Sanidad de Madrid. España. \\ Alfredo Rodríguez Muñoz \\ Psicólogo. Técnico Superior en P.R.L. Personal Investigador \\ Dpto. Psicología Biológica y de la Salud, Facultad de Psicología, \\ Universidad Autónoma de Madrid. España. \\ Correspondencia: \\ Ignacio García Barquero, \\ Joaquin Ruiz Jimenez $102^{\circ} \mathrm{A}$ \\ 28250 Torrelodones (Madrid), España \\ Tels: 650098520/+ $34917434771 /+34918592356$ \\ E-mail: garciabig@munimadrid.es
}

Resumen

Introducción: Es comúnmente aceptado que el grado de satisfacción o bienestar de los trabajadores en su entorno profesional se relaciona de forma directa con la productividad y calidad del servicio prestado. En el caso de los técnicos de drogodependencias los estudios realizados sobre calidad de vida profesional son particularmente escasos, siendo ésta una población sometida a importantes estresores laborales relacionados con la carga emocional de la tarea, tipo de pacientes, necesidad de trabajo en equipo, etc.

Objetivo: El objetivo principal es analizar la relación existente entre la calidad de vida profesional y el desgaste profesional en técnicos de drogodependencias, comparando este grupo profesional con otros profesionales sanitarios que realizan su labor en otros campos y con profesionales no sanitarios.

Material y método: Se ha realizado un estudio transversal y descriptivo en una muestra total de 233 sujetos. El 33\% ( $n=77)$ desarrollan su actividad laboral en el ámbito de las drogodependencias, 79 sujetos (33.3\%) ejercen su profesión en el área sanitaria, y las 77 personas restantes (33\%) pertenecen al grupo de población general trabajadora. Todos ellos contestaron de forma anónima el Cuestionario de Calidad de Vida Profesional (CVP 35) y el Maslach Burnout Inventory (MBI).

Resultados y conclusiones: Los profesionales de drogodependencias presentan menores puntuaciones de de calidad de vida profesional y mayores de desgaste profesional que otros grupos profesionales, siendo ésta diferencia significativa respecto al grupo de trabajadores no sanitarios. Aspectos de la calidad de vida profesional tales como la carga de trabajo, la motivación intrínseca y el apoyo directivo tienen efectos directo 
sobre algunos de los componentes del desgaste profesional, confirmando la importancia de la misma en la prevención de un riesgo psicosocial del trabajo como es el desgaste profesional.

(Med Segur Trab (Internet) 2009; 55 (217): 12-26)

Palabras clave: Calidad de vida profesional, desgaste profesional, técnicos drogodependencias.

Abstract

Introduction: It is widely known that employees' job satisfaction and well-being is related to its productivity and quality of work. In the case of drug dependence technicians, existing research about its professional quality of life is scarce, being this a group exposed to several job stressors linked to emotional workload, patients, work team, etc.

Aim: The main purpose is to analyze the relationship between professional quality of life and burnout in drug dependence technicians, compared to other sanitary and no sanitary professionals groups.

Material and method: A descriptive and cross-sectional study was carried out in a sample of $233.33 \%$ ( $n=77)$ are workers of drug dependence sector, 79 subjects work at sanitary area, and the rest of the sample $(n=77)$ are general working population. All participants completed anonymously the Professional Quality of Life Questionnaire and the Maslach Burnout Inventory.

Results and conclusions: Drug dependence professionals showed lower professional quality of life scores and higher of burnout than other groups. Several aspects of professional quality of life such as workload, intrinsic motivation, and supervisor support showed a main effect on burnout, confirming its importance in preventing burnout.

(Med Segur Trab (Internet) 2009; 55 (217): 12-26)

Keywords: Professional quality of life, Burnout, Drug dependence technicians 


\section{INTRODUCCIÓN}

El grado de satisfacción o bienestar que los profesionales tienen con su trabajo se considera un condicionante básico de la productividad y la calidad de los servicios sanitarios, siendo, a su vez, la satisfacción laboral uno de los principales objetivos de estudio dentro de las organizaciones sanitarias, con la finalidad de evaluar su calidad global. Con el transcurso del tiempo el abordaje de la calidad en las organizaciones sanitarias ha experimentado variaciones, poniéndose de manifiesto la necesidad de orientar el servicio hacia el concepto de calidad total ${ }^{1}$.

La satisfacción profesional ha de contemplarse dentro de la calidad de la organización, a modo de rasgo estructural que favorece el buen funcionamiento y la calidad del servicio prestado, y su inclusión entre los atributos de la gestión de calidad se deriva de la influencia que tiene la satisfacción profesional en la calidad del trabajo realizado $^{2}$. La satisfacción y el bienestar de los trabajadores están estrechamente ligados a dos conceptos como son la calidad de vida profesional y el síndrome de desgaste profesional o burnout. Ambos valoran dimensiones diferentes pero estrechamente relacionadas, como han puesto de manifiesto diversos estudios, y con ellos se pueden identificar elementos clave de la gestión de personas que pueden mejorar su calidad de vida profesional y consecuentemente la calidad de los servicios que prestan a la comunidad.

La calidad de vida profesional es definida por algunos autores ${ }^{3}$ como la experiencia de bienestar derivada del equilibrio que percibe el individuo entre las demandas o carga de trabajo desafiante, intenso y complejo, y los recursos, psicológicos, organizacionales y relacionales de que dispone para afrontar esas demandas. Aunque se dispone de diferentes instrumentos para cuantificarla, son escasos en nuestro país los estudios realizados con el objetivo de medirla entre los diferentes trabajadores.

Por su parte, el desgaste profesional, ha sido habitualmente definido en función de las dimensiones evaluadas por un instrumento de medida, el Maslach Burnout Inventory ${ }^{4,5}$ como un conjunto de síntomas que consisten en agotamiento emocional, despersonalización y falta de realización personal, que puede ocurrir entre individuos que trabajan con personas. Éste síndrome se ha relacionado con profesionales de ayuda que mantienen una relación constante y directa con otras personas y con factores ligados a la organización del trabajo. Desde finales de los años $80^{6,7}$ se conoce la mayor prevalencia de este problema entre los profesionales sanitarios respecto de otros y se ha constatado su elevada incidencia en trabajadores sociales y profesionales de atención a drogodependientes y a la infancia en situación de riesgo y exclusión ${ }^{8-12}$.

El personal sanitario, concretamente, se enfrenta a situaciones laborales que inciden tanto en su calidad de vida profesional como en el riesgo de desgaste. Insatisfacción salarial, problemas de relación con la población atendida, ambigüedad y conflicto de rol, ausencia de reconocimiento social de la profesión y de criterios definidos que posibiliten la valoración de logros, reducidas posibilidades de promoción y el volumen y carga de trabajo, son entre otras, algunas de las causas que se han relacionado en mayor medida con los problemas laborales de este colectivo ${ }^{13}$. Además, son muchos los factores sociales, tecnológicos y económicos que obligan a la exigencia creciente de una mayor dedicación en los profesionales sanitarios, sin que de forma paralela se considere en igual medida aspectos relativos a la calidad de vida profesional.

Respecto al profesional que atiende a personas drogodependientes, su actividad laboral implica el manejo de niveles de frustración de alguna manera más intensos que con otro tipo de pacientes, ya que el porcentaje de recaídas por la naturaleza misma de la enfermedad tiende a ser más alto y el nivel de dificultad de su tratamiento es también elevado. La atención a este tipo de pacientes provoca contratrasferencias muchas veces difíciles de manejar, como señala De las Cuevas ${ }^{13}$. Las características de la personalidad adictiva como pueden ser la dependencia, la pasividad, la poca diferenciación, el temor al rechazo, la dificultad de reconocer y el miedo a expresar emociones, la impulsividad y 
el uso de mecanismos de defensa muy primitivos, como son la negación y la proyección, hacen del tratamiento de estos pacientes una tarea realmente difícil, que puede llegar a ser emocionalmente agotadora.

Existen ya muy diversos trabajos que estudian la calidad de vida profesional y el burnout en el personal sanitario tanto a nivel ambulatorio como hospitalario, nacionales e internacionales pero son menos los que relacionan ambas cuestiones y escasean los referidos al personal dedicado al manejo del paciente drogodependiente. Destacan los trabajos acerca del burnout realizados) en este grupo profesional ${ }^{14}$, en educadores sociales $^{15}$ y en personal voluntario ${ }^{16}$, así como el trabajo sobre clima laboral percibido y determinantes de la satisfacción laboral de Nabitz et al. ${ }^{17}$.

Mas allá de los estudios con diferentes profesionales, en la actualidad nos encontramos con una línea de investigación que centra su atención en los estudios comparativos, destacando en ella los trabajos que comparan profesionales de diferentes países ${ }^{18,19}$, mujeres en contexto de trabajo y no trabajo ${ }^{20}$, enfermeras de atención primaria y especializada $^{21}$, médicos y profesionales de la enseñanza ${ }^{22}$ y profesionales de oncología en diferentes unidades ${ }^{23}$. El presente trabajo tiene como objetivo general efectuar una evaluación de la calidad de vida profesional y el síndrome de burnout en una muestra de técnicos en drogodependencias, realizando un análisis comparativo de los resultados con dos muestras poblacionales de control compuestas, una, por profesionales sanitarios que no realizan su labor en el campo de las drogodependencias y otra, por otros profesionales no sanitarios (población general activa) con un tamaño equivalente, en orden a establecer las similitudes o diferencias entre las mismas.

\section{MATERIAL Y MÉTODO}

La muestra está formada por 233 sujetos de la Comunidad de Madrid y otras Comunidades Autónomas distribuidos en tres grupos compuestos por profesionales que trabajan en drogodependencias, otros profesionales sanitarios (de atención primaria, hospitalaria y geriátrica) y profesionales no sanitarios (población general activa). La edad media es de 42.92 años con una desviación típica de 8.89 y un rango de 24 a 76 años. El 36.1\% de la muestra son hombres. La mayoría de la muestra tiene un contrato laboral fijo (37.5\%) o es funcionario (29.7\%). La distribución en cuanto a área laboral es la siguiente: 77 personas (33\%) desarrollan su actividad laboral en el ámbito de las drogodependencias, 79 sujetos (33.3\%) ejercen su profesión en el área sanitaria, y las 77 personas restantes (33\%) pertenecen al grupo de población general trabajadora.

Se elaboró un protocolo de recogida de datos que fue entregado en papel impreso o en formato digital a los profesionales interesados en su cumplimentación, donde se recogen las diversas variables sociodemográficas y los cuestionarios CVP-35 y MBI. Se buscaban muestras con un tamaño equivalente y no se trabajó con una población fija predeterminada, lo que impide calcular tasas de respuesta, como se ha hecho en otros trabajos relacionados. Se solicitó permiso a los responsables de los centros participantes y se garantizó la participación voluntaria, confidencialidad y anonimato en la recogida y tratamiento de los datos.

El Cuestionario de Calidad de Vida Profesional (CVP-35) ${ }^{24}$ es una medida multidimensional de la calidad de vida profesional. con categorías de respuesta desde "nada" (valor 0), hasta "mucho" (valor 10). Consta de tres dimensiones: apoyo directivo, cargas de trabajo, y motivación intrínseca, y proporciona una medida global de la percepción de la calidad de vida profesional.

El Maslach Burnout Inventory-Human Services Survey (MBI-HSS) ${ }^{5}$. Consta de 22 ítems y en él se pueden diferenciar tres subescalas: agotamiento emocional (AE), despersonalización (DP) y realización personal (RP). Emplea un sistema de respuestas que puntúan la frecuencia con que el sujeto experimenta los sentimientos enunciados en 
cada ítem en una escala tipo Likert, que van desde "nunca" (0) hasta "diariamente" (6). La relación entre puntuación y grado de burnout es dimensional. Es decir, no existe un punto de corte que indique se existe o no existe tal entidad. Se distribuyen los rangos de las puntuaciones totales de cada subescala en tres tramos que definen un nivel de burnout experimentado como Bajo ( $\mathrm{AE}=\leq 14 ; \mathrm{DP}=\leq 3 ; \mathrm{RP}=\geq 40$ ), Medio ( $\mathrm{AE}=15-24 ; \mathrm{DP}=4-9$; $\mathrm{RP}=33-39)$ o Alto $(\mathrm{AE}=\geq 25 ; \mathrm{DP}=\geq 10 ; \mathrm{RP}=\leq 32)$.

\section{RESULTADOS}

Se realizó un análisis descriptivo de las características sociodemográficas en función del tipo de área de trabajo (Tabla 1), mediante la prueba estadística análisis de varianza (ANOVA) y la prueba de Scheffé. En el análisis univariante, los profesionales de drogodependencias presentaban mayores niveles de burnout que el resto de los trabajadores. En concreto, presentaron mayor agotamiento emocional ( $p<0,05)$ que los trabajadores de población general, no encontrándose diferencias significativas con los trabajadores sanitarios. Del mismo modo, informaron de mayores niveles de despersonalización comparados con los trabajadores de población general $(\mathrm{p}<0,01)$ y mayores niveles de realización personal, aunque las diferencias en esta última variable no resultaron significativas $(\mathrm{p}=0.106)$.

Por otra parte, los profesionales de drogodependencias también mostraban peores indicadores de calidad de vida profesional que el resto de trabajadores. Las diferencias se plantean respecto al grupo de población general trabajadora, puesto que con el grupo de sanitarios no se hallaron diferencias significativas. Específicamente, comparado con el grupo de población general, los trabajadores de drogas informaron tener menos apoyo directivo $(\mathrm{p}<0,05)$, y mayores niveles de sobrecarga de trabajo $(\mathrm{p}<0,01)$.

Para comprobar el efecto de los factores sociodemográficos sobre las variables del estudio, se realizó un análisis de modelo lineal general multivariante (MANOVA). Se tomaron como variables dependientes cada uno de los componentes del burnout (agotamiento emocional, despersonalización, realización personal) y de la calidad de vida laboral (apoyo directivo, cargas de trabajo, motivación intrínseca). Como variables independientes se utilizaron diversas características sociodemográficas comunes a los distintos grupos laborales (género, edad, tipo de contrato y sector laboral). El criterio para determinar las diferencias significativas al nivel multivariado fué Lambda de Wilks y para identificar los niveles de las variables independientes que difieren entre si, se empleó la prueba de Scheffé, que requiere una mayor diferencia entre las medias para la significación. 
Tabla 1 - Análisis descriptivo de las variables del estudio según el área de trabajo

\begin{tabular}{|c|c|c|}
\hline Variables & Media & D.T. \\
\hline \multicolumn{3}{|l|}{ Agotamiento emocional } \\
\hline Área Drogas & 21,0 & 7,7 \\
\hline Área Sanitaria & 18,4 & 7,9 \\
\hline Poblacion general trabajadora & 18,1 & 7,6 \\
\hline Muestra Total & 19,2 & 7,8 \\
\hline \multicolumn{3}{|l|}{ Despersonalización } \\
\hline Área Drogas & 9,1 & 4,1 \\
\hline Área Sanitaria & 7,9 & 3,6 \\
\hline Población general trabajadora & 7,0 & 3,6 \\
\hline Muestra Total & 8,0 & 3,8 \\
\hline \multicolumn{3}{|l|}{ Realización personal } \\
\hline Área Drogas & 27,1 & 6,4 \\
\hline Área Sanitaria & 26,6 & 8,1 \\
\hline Población general trabajadora & 24,7 & 7,4 \\
\hline Muestra Total & 26,1 & 7,4 \\
\hline \multicolumn{3}{|l|}{ Apoyo directivo } \\
\hline Área Drogas & 65,3 & 18,6 \\
\hline Área Sanitaria & 69,7 & 18,2 \\
\hline Población general trabajadora & 73,1 & 17,3 \\
\hline Muestra Total & 69,4 & 18,2 \\
\hline \multicolumn{3}{|l|}{ Cargas de trabajo } \\
\hline Área Drogas & 65,1 & 13,4 \\
\hline Área Sanitaria & 62,8 & 14,4 \\
\hline Población general trabajadora & 56,8 & 14,7 \\
\hline Muestra Total & 61,6 & 14,5 \\
\hline \multicolumn{3}{|l|}{ Motivación intrínseca } \\
\hline Área Drogas & 62,7 & 9,5 \\
\hline Área Sanitaria & 66,1 & 12,6 \\
\hline Población general trabajadora & 62,4 & 10,2 \\
\hline Muestra Total & 63,7 & 11,0 \\
\hline \multicolumn{3}{|l|}{ Calidad de vida profesional } \\
\hline Área Drogas & 193,1 & 27,8 \\
\hline Área Sanitaria & 198,6 & 23,9 \\
\hline Población general trabajadora & 192,3 & 28,4 \\
\hline Muestra Total & 194,7 & 26,8 \\
\hline
\end{tabular}

Los resultados del análisis multivariado confirman que existen efectos principales multivariados de la variable sector laboral o profesión $\left(\mathrm{F}_{4,228}=2.071\right)$. Según la prueba deScheffé,considerandola profesión desempeñada,losprofesionalesdedrogodependencias 
presentan mayores niveles de cargas de trabajo $\left(M_{\text {Drogas }}=65,09\right)$, existiendo diferencias significativas $(\mathrm{p}<0,05)$ con los trabajadores de población general $\left(M_{\text {General }}=56,80\right)$. También se apreciaron diferencias significativas $(\mathrm{p}<0,05)$ entre los trabajadores del área sanitaria y los de población general, presentando estos últimos menores niveles de carga de trabajo. Los trabajadores del área de drogas presentaban mayores niveles de despersonalización $\left(M_{\text {Drogas }}=9,09\right)$, existiendo claras diferencias significativas $(\mathrm{p}<0,01)$ con los trabajadores de población general $\left(M_{\text {General }}=7,00\right)$. No se encontraron diferencias significativas entre el resto de los grupos. En cuanto al agotamiento emocional se observó una tendencia, aunque no significativa.

Tabla 2 - Resultado del contraste de la prueba de Scheffé

\begin{tabular}{|c|c|c|c|c|}
\hline & Media & D.T. & Sanitarios & General \\
\hline \multicolumn{5}{|l|}{ Cargas de trabajo } \\
\hline Área Drogas & 65,1 & 13,4 & n.s. & $8,36^{*}$ \\
\hline Sanitarios & 62,8 & 14,4 & & $6,08^{*}$ \\
\hline Población general trabajadora & 56,8 & 14,7 & & \\
\hline \multicolumn{5}{|l|}{ Despersonalización } \\
\hline Área Drogas & 9,1 & 4,1 & n.s. & $2,09^{* *}$ \\
\hline Sanitarios & 7,9 & 3,6 & & n.s. \\
\hline Población general trabajadora & 7,0 & 3,6 & & \\
\hline \multicolumn{5}{|l|}{ Agotamiento emocional } \\
\hline Área Drogas & 20,96 & 7,65 & n.s. & $2,84 \dagger$ \\
\hline Sanitarios & 18,41 & 7,85 & & n.s. \\
\hline Población general trabajadora & 18,11 & 7,59 & & \\
\hline$\dagger \mathrm{p}<.0,10 ; * * \mathrm{p}<0,01, * \mathrm{p}<0,05$ & & & & \\
\hline
\end{tabular}

Para profundizar en el análisis del desgaste profesional se procedió a la comparación entre grupos poblacionales en función de los niveles del síndrome (bajo, medio, alto). Se ha comprobado que existen diferencias en las puntuaciones medias en despersonalización y agotamiento emocional, pero también resulta interesante comprobar si existen diferencias en los niveles. En la tabla 3 aparecen los niveles de burnout por área profesional.

Tabla 3 - Comparación de prevalencia del burnout en función de los distintos grupos poblacionales

\begin{tabular}{lccccccccc}
\hline & \multicolumn{3}{c}{ Área Drogas } & & \multicolumn{3}{c}{ Área Sanitaria } & \multicolumn{3}{c}{ Población General } \\
\hline & AE & DP & RP & AE & DP & RP & AE & DP & RP \\
Niveles Altos & 35,1 & 45,5 & 80,5 & 15,2 & 29,1 & 78,5 & 19,5 & 22,1 & 85,7 \\
Niveles Medios & 44,2 & 48,1 & 19,5 & 51,9 & 58,2 & 12,7 & 48,1 & 61,0 & 11,7 \\
Niveles Bajos & 20,8 & 6,5 & 0 & 32,9 & 12,7 & 8,9 & 32,5 & 16,9 & 2,6 \\
\hline
\end{tabular}

AE: Agotamiento Emocional; DP: Despersonalización; RP: Falta de realización personal en el trabajo.

Como puede observarse, las tasas de severidad difieren significativamente en función del área de trabajo. Siguiendo los análisis de la prueba de chi-cuadrado, los profesionales que trabajan en drogodependencias presentan mayor prevalencia de niveles altos de agotamiento emocional, comparados con los otros grupos $(\mathrm{p}<0,05)$. Del mismo 
modo, los trabajadores del área de drogas presentan mayores tasas de despersonalización $(\mathrm{p}<0.05)$. En cuanto a la realización personal, los trabajadores de población general presentan niveles más altos de realización general $(\mathrm{p}<0,05)$.

Para analizar las relaciones existentes entre el burnout y la calidad de vida profesional, en primer lugar se realizó un análisis de correlación bivariada de Pearson. Como se aprecia en la tabla 4, los análisis de correlación indican, tal y como se esperaba, que existe una elevada asociación entre los distintos componentes del desgaste profesional o burnout, y entre las dimensiones que conforman la calidad de vida profesional. Por otra parte, las relaciones entre el burnout y la calidad de vida profesional son negativas $y$ significativas.

Tabla 4 - Matriz de correlaciones entre todas las variables $(\mathrm{N}=\mathbf{2 3 3})$

\begin{tabular}{|c|c|c|c|c|c|c|}
\hline Variable & $\mathbf{1}$ & 2 & 3 & 4 & 5 & 6 \\
\hline 1. Agotamiento emocional & --- & & & & & \\
\hline 2. Despersonalización & $0,47^{* * *}$ & --- & & & & \\
\hline 3. Realización personal & $-0,26^{* * *}$ & $-0,38^{* * *}$ & -- & & & \\
\hline 4. Apoyo Directivo & $-0,38^{* * *}$ & $-0,36^{\text {**** }}$ & $0,52^{\text {***k }}$ & --- & & \\
\hline 5. Cargas de trabajo & $0,50^{* * *}$ & $0,40^{* *}$ & $-0,19 * *$ & $-0,25^{* * *}$ & --- & \\
\hline 6. Motivación Intrínseca & $-0,33^{* * *}$ & $-0,37^{* * *}$ & $0,60^{* * *}$ & $0,60^{\text {**** }}$ & $-0,16^{* * *}$ & -- \\
\hline 7. Calidad vida profesional global & $-0,12$ & $-0,18^{\text {*k }}$ & $0,49^{\text {***k }}$ & $0,78^{* * *}$ & $0,30^{* * *}$ & $0,73^{\text {*⿻丷木 }}$ \\
\hline
\end{tabular}

Según los modelos transaccionales el burnout es resultando de la combinación de los estresores, ya sean laborales o sociales, y de su interacción con ciertas características individuales ${ }^{25,26}$. De acuerdo con este planteamiento, resulta sensato plantear que la calidad de vida profesional influya en el burnout. Para ello, se realizaron diversos análisis de regresión múltiple jerárquica por pasos, para determinar los efectos principales y de interacción de las variables de calidad de vida profesional (apoyo directivo, cargas de trabajo, y motivación intrínseca). Se estableció una probabilidad asociada a $\mathrm{F}<0,05$ para la entrada de las variables independientes, junto con una probabilidad de salida mayor de 0,10. Se siguió el procedimiento recomendado por Cohen, Cohen, West y Aiken $(2003)^{27}$. Para minimizar los efectos de la multicolinealidad, y se realizaron todos los análisis de regresión con las variables independientes estandarizadas. Las variables independientes entraron en la ecuación de regresión en tres pasos sucesivos. En primer lugar, se introdujo la variables sociodemográfica que había sido significativa en el MANOVA, es decir, el área de trabajo. En el segundo paso, se introdujeron las variables de calidad de vida profesional: el apoyo directivo, las cargas de trabajo, y la motivación intrínseca. Finalmente, en el tercer paso se introdujeron las dos variables que resultan de la interacción (Cargas de trabajo X Apoyo directivo, Cargas de trabajo X Motivación intrínseca). Como variable dependiente se utilizaron los tres componentes del burnout: agotamiento emocional, despersonalización y realización personal. Previo a los resultados se comprobaron los supuestos del modelo; independencia, homocedasticidad y la no colinealidad. En primer lugar, el estadístico Durbin-Watson nos informó de la independencia de los residuos, ya que los valores del modelo de regresión se encontraban dentro del rango recomendado $(1,5-2,5)$ para considerar independientes las observaciones ${ }^{28}$. Por su parte, el diagrama de dispersión de los pronósticos tipificados por los residuos tipificados nos indicó la igualdad de varianzas. Respecto a los estadísticos de colinealidad, de acuerdo con Kleinbaum, Kupper y Mueller (1988) ${ }^{29}$, índices de inflación mayores de 10 y valores de tolerancia menores de 0,10 pueden indicar la presencia de multicolinealidad. Los resultados de estos dos índices permitieron descartar la existencia de colinealidad entre las variables independientes consideradas en el estudio. 
Tabla 5 - Análisis de regresión múltiple jerárquica.

\begin{tabular}{|c|c|c|c|}
\hline \multirow[b]{2}{*}{ Pasos del modelo y Variables ${ }^{a}$} & \multicolumn{2}{|c|}{ Agotamiento Emocional } & \multirow[b]{2}{*}{ B paso 3} \\
\hline & B paso 1 & B paso 2 & \\
\hline \multicolumn{4}{|l|}{ Variable control } \\
\hline Área de trabajo & $-0,13^{*}$ & $-0,02$ & $-0,02$ \\
\hline \multicolumn{4}{|l|}{ Efectos principales } \\
\hline Cargas de trabajo & & $0,43^{* *}$ & $0,46^{* * *}$ \\
\hline Apoyo directivo & & $-0,16^{*}$ & $-0,14^{*}$ \\
\hline Motivación intrínseca & & $-0,16^{*}$ & $-0,21^{* *}$ \\
\hline \multicolumn{4}{|l|}{ Efectos de interacción } \\
\hline Cargas trabajo X Apoyo directivo & & & $-0,05$ \\
\hline Cargas trabajo $\mathrm{X}$ Motivación intrínseca & & & $-0,06$ \\
\hline $\mathrm{R}^{2}$ & 0,02 & 0,36 & 0,37 \\
\hline$\Delta \mathbf{R}^{2}$ & $0,02 *$ & $0,34^{* * * * *}$ & 0,01 \\
\hline
\end{tabular}

\begin{tabular}{|c|c|c|c|}
\hline \multicolumn{4}{|c|}{ Despersonalización } \\
\hline Variable control & & & \\
\hline Área de trabajo & $-0,22^{* *}$ & $-0,13^{*}$ & $-0,15^{*}$ \\
\hline \multicolumn{4}{|l|}{ Efectos principales } \\
\hline Cargas de trabajo & & $0,29^{* * * * *}$ & $0,33^{* * *}$ \\
\hline Apoyo directivo & & $-0,1$ & $-0,06$ \\
\hline Motivación intrínseca & & $-0,27^{\text {***** }}$ & $-0,35^{\text {**** }}$ \\
\hline \multicolumn{4}{|l|}{ Efectos de interacción } \\
\hline Cargas trabajo X Apoyo directivo & & & 0,04 \\
\hline Cargas trabajo $\mathrm{X}$ Motivación intrínseca & & & $-0,18^{*}$ \\
\hline $\mathrm{R}^{2}$ & 0,05 & 0,29 & 0,31 \\
\hline \multirow[t]{2}{*}{$\Delta \mathrm{R}^{2}$} & $0,05^{* *}$ & $0,24^{* * * * *}$ & $0,02^{*}$ \\
\hline & \multicolumn{3}{|c|}{ Realización personal } \\
\hline \multicolumn{4}{|l|}{ Variable control } \\
\hline Área de trabajo & $.13^{*}$ & $.20 * * *$ & $.18^{* * * * *}$ \\
\hline \multicolumn{4}{|l|}{ Efectos principales } \\
\hline Cargas de trabajo & & $-.10 \dagger$ & $-.10 \dagger$ \\
\hline Apoyo directivo & & $.28^{* * * *}$ & $.27^{* * * * * *}$ \\
\hline Motivación intrínseca & & $.40^{* * * *}$ & $.44^{* * * * *}$ \\
\hline \multicolumn{4}{|l|}{ Efectos de interacción } \\
\hline Cargas trabajo $\mathrm{X}$ Apoyo directivo & & & $-.13 \dagger$ \\
\hline Cargas trabajo X Motivación intrínseca & & & $.12 \dagger$ \\
\hline \multicolumn{4}{|l|}{$\mathrm{R}^{2}$} \\
\hline$\Delta \mathrm{R}^{2}$ & .02 & .46 & .31 \\
\hline
\end{tabular}


Tal y como se aprecia en la tabla 5, en la variable agotamiento emocional se observa un efecto principal positivo de cargas de trabajo $(\beta=0,17$; $<<0,01)$, y negativo de la motivación intrínseca $(\beta=-0,21 ; \mathrm{p}<0,01)$, y del apoyo directivo $(\beta=-0,14 ; \mathrm{p}<0,05)$. Respecto a los efectos de interacción, los resultados muestran que ninguna de las interacciones resulta significativa. Respecto a la despersonalización se aprecia un efecto directo de cargas de trabajo $(\beta=0,33 ; \mathrm{p}<0,01)$, y negativo del área de trabajo $(\beta=-0,15$; $\mathrm{p}<0,05)$, motivación intrínseca $(\beta=-0,35 ; \mathrm{p}<0,05)$, y en relación a los efectos de interacción, se observa que la motivación intrínseca modera la relación entre cargas de trabajo y despersonalización $(\beta=-0,18 ; p<0,05)$. En la variable realización personal se aprecia que las variables que tienen un efecto directo son el área de trabajo $(\beta=0,18 ; \mathrm{p}$ $<0,01)$, apoyo directivo $(\beta=0,27 ; \mathrm{p}<0,01)$, y motivación intrínseca $(\beta=0,44 ; \mathrm{p}<0,01)$. Respecto a los efectos de interacción, se observa que dos tendencias significativas del apoyo directivo $(\beta=-0,13 ; \mathrm{p}<0,10)$ y la motivación intrínseca $(\beta=0,12 ; \mathrm{p}<0,10)$ en la relación entre cargas de trabajo y realización personal.

Para profundizar en la interpretación de estos resultados se ha representado gráficamente las interacciones, de tal manera que el valor alto o bajo en una variable corresponde a puntuaciones que están una desviación típica por encima o por debajo de la media $^{27}$. La figura 1 muestra que aquellos sujetos con bajos niveles de motivación presentan mayores niveles de despersonalización y la figura 2 muestra que aquellos sujetos con bajos niveles de motivación presentan menor realización personal. Al contrario, aquellos sujetos con alto apoyo directivo presentan mayores niveles de realización personal (figura 3).

Figura 1 - Interacción de cargas de trabajo X motivación intrínseca

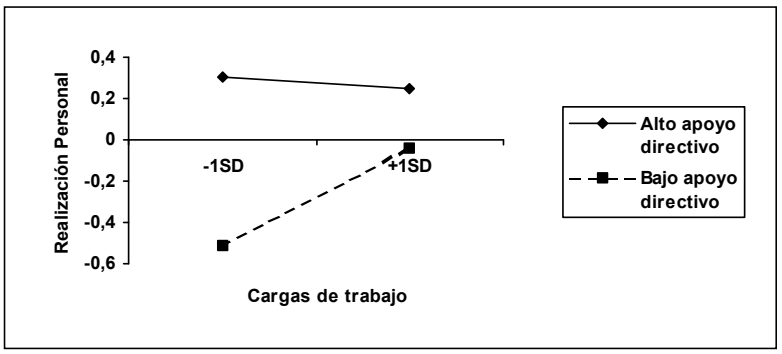

Figura 2 - Interacción de cargas de trabajo X motivación intrínseca

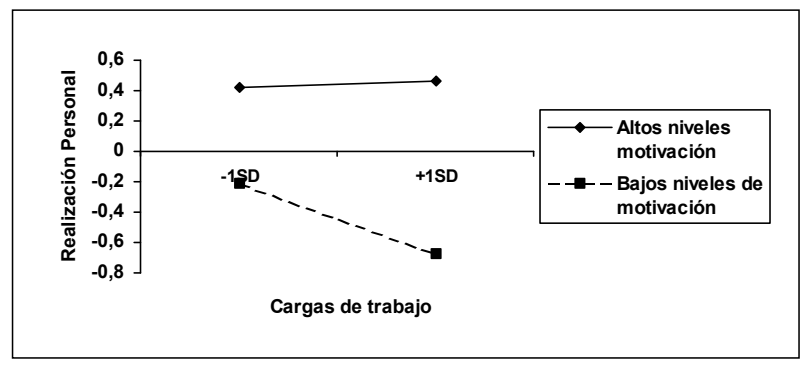

Figura 3. Interacción de cargas de trabajo X realización personal

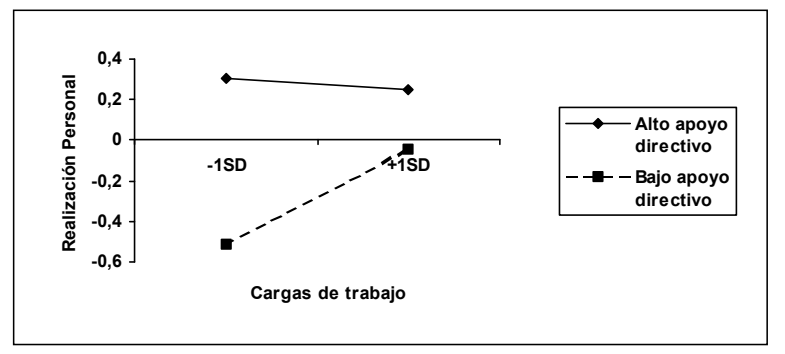




\section{DISCUSIÓN.}

El objetivo de la presente investigación era analizar la relación existente entre la calidad de vida profesional y el síndrome de burnout en una muestra de técnicos en drogodependencias, comparando este grupo profesional con otros profesionales sanitarios que no realizan su labor en el campo de las adicciones y con personal no sanitario (población general activa).

En el análisis descriptivo de las variables estudiadas en el presente estudio, en concreto en el análisis univariante, los profesionales de drogodependencias presentaban mayores niveles de desgaste profesional que el resto de los trabajadores, en concreto mayor agotamiento emocional y despersonalización; sin embargo, y esto es un primer dato de interés, las diferencias no fueron significativas con los trabajadores sanitarios. De la misma manera, los trabajadores del ámbito de las drogodependencias, mostraban peores indicadores de calidad de vida profesional, pero de nuevo estas diferencias no fueron significativas respecto del grupo de sanitarios. Estos datos nos hacen pensar que si bien el desgaste profesional es un riesgo psicosocial del trabajo compartido por los profesionales sanitarios y de drogodependencias, es necesario profundizar en sus diferentes condiciones de trabajo y aspectos específicos para encontrar diferencias significativas entre ambas muestras.

El análisis multivariado permitió comprobar mayores niveles de carga de trabajo en los profesionales de drogodependencias con claras diferencias significativas respecto de los trabajadores de población general, diferencias que también lo eran en los trabajadores sanitarios respecto de aquellos, confirmando asimismo los resultados que respecto de la despersonalización y agotamiento emocional se habían producido en los diferentes grupos. Estos datos coinciden con trabajos exploratorios anteriores en profesionales de drogodependencias ${ }^{14}$, donde se apuntaba la presencia de malestar profesional en el colectivo que atiende a personas con problemas de abuso o dependencia de sustancias, en la línea que se ha venido observando en los estudios que al respecto se han realizado en diferentes equipos de atención sanitaria en otros niveles de atención ${ }^{30-32}$. Es interesante señalar, que en los trabajadores de drogodependencias los niveles altos de agotamiento emocional tienen una mayor prevalencia, hecho que también ha sido valorado en otros estudios $^{13}$. Hay que hacer notar que la dimensión de agotamiento es sobre la que hay más acuerdo como punto clave del concepto de burnout ${ }^{6,7}$ y es la dimensión que guarda mayor relación con consecuencias físicas negativas en la salud de los trabajadores ${ }^{33}$. En lo referente a la realización personal, los resultados más independientes de esta variable, confirman los nuevos planteamientos en la evaluación del síndrome que la señalan como un constructo independiente del burnout que puede estar actuando como un estilo de afrontamiento o un consecuente del proceso de burnout ${ }^{34}$.

El análisis correlacional, confirma como se esperaba una elevada correlación significativa y negativa entre los distintos componentes del burnout y las dimensiones que conforman la calidad de vida profesional, hecho que ha sido también observado en otro estudio realizado en Atención Primaria ${ }^{35}$. Esta relación entre ambos constructos señala que cuando los trabajadores perciben una alta calidad de vida profesional, este hecho se relaciona con un menos desgaste profesional autoinformado. De forma específica, si existe apoyo directivo y la persona se siente motivada en su trabajo, se relatan menores niveles de agotamiento, si además se percibe una baja sobrecarga laboral, existirán menores actitudes de despersonalización frente a los usuarios del servicio y mayor realización profesional en el trabajo. En ésta línea, algunos autores ${ }^{36}$ recuerdan la necesidad de introducir modificaciones en las condiciones de trabajo y en la organización empresarial en función de las variables determinantes de la satisfacción y encuentran diferencias en los resultados respecto de las variables sociodemográficas, circunstancia que no es valorada por el resto de los autores citados como significativa y en el presente estudio lo es tan solo en el área de trabajo, como queda descrito. El análisis de regresión permite observar un efecto principal positivo de las cargas de trabajo sobre la variable agotamiento emocional y negativo de la motivación intrínseca y del apoyo directivo; en 
la variable despersonalización un efecto directo de cargas de trabajo y negativo del área de trabajo y la motivación intrínseca. En el estudio realizado en el centro de adicciones Jellinek en los Países Bajos, la presión asistencial y/o organizativa resultó ser la que de manera más clara influye en la aparición de agotamiento emocional en los distintos profesionales ${ }^{17}$. Tal y como han descrito estudios previos, la sobrecarga laboral se relaciona de forma positiva con el burnout ${ }^{37}$. En este sentido, autores como Atance (1997) ${ }^{38}$ hacen referencia a la relación existente entre el burnout y la sobrecarga laboral en profesionales asistenciales, por lo cual este aspecto produciría una disminución de la calidad del trabajo tanto cualitativa como cuantitativamente.

Se han observado interesantes efectos de interacción, de manera que, por un lado, la motivación intrínseca modera la relación entre cargas de trabajo y despersonalización. En un estudio de clima laboral realizado por el Instituto de Adicciones de la ciudad de Madrid $^{39}$ se comprobó como los profesionales de drogodependencias mostraban un nivel elevado de compromiso con su trabajo; es posible que esta alta motivación intrínseca funcione en este grupo profesional de forma compensadora frente a las variables que, como se ha descrito, se relacionan positivamente con su agotamiento emocional y despersonalización. Por otro lado, respecto a la variable de realización personal percibida por los sujetos de la muestra, se aprecia un efecto directo de área de trabajo, apoyo directivo y motivación intrínseca mostrando de nuevo un efecto de interacción, observando dos tendencias significativas del apoyo directivo y la motivación intrínseca en relación con cargas de trabajo y realización personal, resultando que aquellos sujetos con baja motivación presentan menores niveles de realización personal y de forma contraria aquellos sujetos con alto apoyo directivo presenten mayores niveles de realización personal. Diversos autores consideran que la ausencia de apoyo significativo es un factor crítico en el desarrollo del burnout particularmente para los trabajadores más aislados profesionalmente ${ }^{40}$. En cierta medida, los trabajadores de drogodependencias, por las características específicas de su labor asistencial, por su organización dentro de la estructura sanitaria y por cuestiones de carácter sociológico, en ocasiones pueden percibir esta sensación de aislamiento, hecho que podría relacionarse con los resultados expuestos. Hay que resaltar que los efectos descritos tienen un valor predictivo, por lo que muy probablemente se mantendrían de aumentar las muestras poblacionales y podrían considerarse como una característica o rasgo de las mismas, de ahí su valor añadido.

Analizando conjuntamente los aspectos expuestos, que representan en síntesis los hallazgos resultantes del presente análisis, se apuntan datos que permiten ciertas reflexiones. En primer lugar y en referencia a los diversos estudios arriba citados, y realizados en el ámbito sanitario, los resultados que allí se encuentran, en concreto las variables de relación entre los diferentes componentes de la calidad de vida profesional y el grado de desgaste profesional, no difieren en su acepción genera respecto de los nuestros, aunque hay que tener en cuenta que la metodología del presente estudio, con comparación de muestras, es diferente y allí se trata de estudios descriptivos sobre una misma muestra poblacional; sin embargo la relación entre las variables de la calidad de vida profesional y el burnout en las muestras tanto de técnicos de drogodependencias, como de sanitarios, no es muy distinta de la que describen aquellos. De otro lado, la ausencia de diferencia significativas en cuanto a los niveles de burnout y calidad de vida profesional percibida entre los grupos de profesionales de drogodependencias y de sanitarios, hace que los profesionales que ejercemos en el terreno de aquellas y las Instituciones que nos albergan, podamos de alguna manera aprender de la experiencia y conocimientos derivados de los diferentes estudios que sobre ambas cuestiones se han llevado a cabo tanto en la asistencia primaria como en la hospitalaria, colectivos con los que trabajamos y nos relacionamos a diario.

La asistencia a las adicciones experimenta cambios y progresos día a día y todo cambio precisa de una adaptación y una dosis extra de energía, lo que puede aumentar la sobrecarga del trabajador, pero el enriquecimiento que supone el intercambio de experiencias con colectivos relacionados, es muy probable que lo compensara en parte. Por otra parte los trabajadores de drogodependencias tendrían mucho que decir acerca 
de las estrategias de afrontamiento que utilizan en un trabajo difícil y complicado como es el suyo ${ }^{41,42}$. Diversos autores han llamado la atención sobre la escasa importancia que se ha dado a las variables de la personalidad y al afrontamiento frente a las variables sociodemográficas y laborales dentro del ámbito del burnout (Hernández Zamora et al., 2004). Es muy posible que el presente estudio pudiera verse enriquecido en un futuro con la inclusión de alguna, de las diversas que existen, escala de medición de afrontamiento del estrés, lo que permitiría apuntar resultados de alguna manera más concluyentes.

La realización, en cualquier caso de este tipo de estudios, debería ser práctica tan habitual como lo es la utilización de encuestas de satisfacción del usuario, ya que permitirían conocer más sobre el estado de opinión de los trabajadores en relación con su empresa y con la calidad que perciben en su vida laboral, y ello a su vez haría factible la introducción de mecanismos correctores para prevenir el desgaste profesional que constituye uno de los problemas del actual sistema sanitario público. El presente estudio pretende ser sólo una primera aproximación a estos factores y deberá completarse en un futuro con muestras mayores, así como con aproximaciones procesuales y longitudinales.

Las reformas, aunque tímidas, que se van acometiendo a cabo en atención primaria y que incluyen el desarrollo de la carrera profesional, la desburocratización o las mejoras en las condiciones laborales, son objeto de estudio en la actualidad, con la intención de percibir su impacto en la calidad global de la organización, de la misma manera que lo han sido en otros países como el Reino Unido ${ }^{43}$. Es de desear que éstas y las conclusiones que se puedan hacer de los estudios en curso, sean valoradas por las Instituciones que tengan a su cargo la atención a pacientes drogodependientes, junto con las que ellas puedan hacer de los propios, ya es muy posible que el grado de calidad de vida profesional percibida y el de burnout con ella relacionado no sean, en sus diferentes dimensiones, muy distintos en ambos niveles de atención.

En orden a conseguir una atención de calidad resulta importante un compromiso organizacional para que la calidad de vida profesional sea medida periódicamente y objeto de un proceso de mejora continuo ${ }^{44}$. Los profesionales sanitarios solicitan hoy día más participación con posibilidad de que sus ideas sean escuchadas y aplicadas por unos directivos accesibles y conocedores de la realidad, que reconozcan su esfuerzo y les informen sobre los resultados de su trabajo. El aumento de los recursos, el cambio de estilo directivo y el establecimiento de medidas organizativas eficientes deben ser las prioridades de la organización, la mejora sobre estos aspectos influirá en la salud de los profesionales, en la calidad de la atención prestada y la satisfacción de los usuarios con el sistema.

\section{REFERENCIAS}

1. Dean JW Jr., Bowen DE. Management Theory and Total Quality: Improving Research and Practice through Theory Development. Academy of Management Review 1994; 19: 399-403.

2. Firth-Cozens J. Interventions to improve physicians' well-being and patient-care. Soc Sci Med, 2001; 52: 215-22.

3. García Sanchez S. La qualitat de vida professional com a avantatge competitiu. Revista de Qualita 1993; 11: 4-9.

4. Maslach C, Jackson SE. The measurement of experienced burn-out. Journal of Occupational Behavior 1981; 2: 99-113.

5. Maslach C, Jackson S. Maslach Burnout Inventory ( $2^{a}$ ed.). Palo Alto, California: Consulting Psychologists Press; 1986.

6. Shirom A. Burnout in work organization. En L. Cooper, I. Robertson, (Editors). International review of industrial and organizational psychology (pp. 25-48). Chichester: John Wiley; 1989.

7. Garden AM. Burnout: the effect of psychological type on research findings. Journal of Occupational and Health Psychology 1989; 62: 223-4.

8. Barría. Sindrome de burnout en asistentes sociales del Servicio Nacional de Menores. On line 2002. Disponible en: htpp://psiquiatría.com/artículos/estrés/11687. 
9. McGee RA. Burnout and professional decision making: An analogue study. Journal of Counselling Psychology 1989; 36: 345-351.

10. Lee RT, Ashforth BE. A longitudinal study of burnout among supervisors and managers: comparison between the Leiter and Maslach (1968) and Golembiewsky et al. (1986) Models. Organizational Behavior and Human Decision Processes 1993; 54: 369-398.

11. Stevens JP. Applied multivariate statistics for the social sciences. Mahwah, New Jersey: Lawrence Erlbaum Associates; 2002.

12. Stevens M, Higgins DJ. The influence or risk and protective factors on burnout experienced by those who work with maltreated children. Child Abuse Review 2002; 11: 313-331.

13. De las Cuevas C. Burnout en los profesionales de atención a las drogodependencias. Trastornos Adictivos 2003; 5 (2): 107-13.

14. Pedrero EJ, Puerta C, Lagares A, Sáez A, García Barquero I. Estudio del Burnout en profesionales de drogodependencias. Trastornos Adictivos 2004; 6(1): 5-15.

15. Blanch A, Aluja A, Biscarri J. Síndrome de quemarse en el trabajo (burnout) y estrategias de afrontamiento: un modelo de relaciones estructurales. Revista de Psicología del Trabajo y de las Organizaciones 2002; 18 : 57-74.

16. Chacón F, Vecina ML. Motivaciones y burnout en el voluntariado. Intervención Psicosocial 1999; 8: 31-42.

17. Nabitz U, Zwijnenburg W, van der Voet S. Job satisfaction of the personnel of the addiction center Jellinek, Amsterdam: Register of Alcohol, Drugs and Tobacco Research. Netherland; 2000.

18. Gil-Monte PR, Schaufeli W. Burnout en enfermería: un estudio comparativo España-Holanda. Psicología del Trabajo y las Organizaciones 1991; 19(7): 121-130.

19. Moreno-Jiménez B, Garrosa Hernández E, Benavides-Pereira AM, Gálvez Herrer M. Estudios transculturales del burnout. Los estudios transculturales Brasil - España. Revista Colombiana de Psicología 2003; $12:$ 9-18.

20. Agut S, Grau R, Beas M. Burnout en mujeres un estudio comparativo entre contextos de trabajo y no trabajo. Ponencia presentada en el Simposio “Aspectos psicosociales del estrés ocupacional”. Univesitat Jaume I. 2000.

21. Artiga Ruiz A. La enfermería ante el síndrome de burnout. Estudio comparativo entre atención primaria y atención especializada. Revista Enfermería Científica, 2000; 220-221: 11-16.

22. Barranza Macías A, Carrasco Soto R, Arreola Corral M. Síndrome de burnout: un estudio comparativo entre profesores y médicos de la ciudad de Durango. Universidad Pedagógica de Durango. Investigación Educativa 2007; 6: 63-73.

23. Martín Romero CM, Lopez Lorent RM, Fuentes Gonzalez CI, García García E. Estudio comparativo del síndrome de burnout en profesionales de oncología: incidencia y gravedad. Medicina Paliativa 2005; 7(3): 85-93.

24. Martín J, Cortés JA, Morente M, Caboblanco M, Garito J, Rodríguez A. Características métricas del Cuestionario de Calidad de Vida Profesional (CVP-35). Gac Sanit 2004; 18(2): 129-36.

25. Gil-Monte PR, Peiró JM. Desgaste psíquico en el trabajo: el síndrome de quemarse. Madrid: Síntesis; 1997.

26. Moreno-Jiménez B, Morett N, Rodríguez-Muñoz A, Morante M.E. La personalidad resistente como variable moduladora del síndrome de burnout: un estudio en una muestra de bomberos. Psicothema 2006; 18: 413-418.

27. Cohen J, Cohen P, West SG, Aiken LS. Applied multiple regression/correlation analysis for the behavioral sciences (3rd Ed.). Mahwah, NJ: Erlbaum; 2003.

28. Durbin J, Watson GS. Testing for serial correlation in least squares regression. Biometrika,1971; 58: 1-19.

29. Kleinbaum DG, Kupper LL, Muller K. Applied regression analysis and other multivariate methods. Boston: PWS-Kent; 1988.

30. Fernández Martínez O, Moreno Suárez S, Hidalgo Cabrera C, García del Río B, Martín Tapia A. Calidad de vida profesional de los médicos residentes. Archivos de Medicina 2007; 3(1): 1-14.

31. Fernández AM, Santa E, Casado del Olmo MI. Calidad de vida profesional de los profesionales de enfermería en atención primaria de Soria. Biblioteca Lascasas; 2007.

32. Juvete MT, Lacalle M, Riesgo R, Cortés JA, Mateo C. Estudio de la calidad de vida profesional en los trabajadores de atención primaria del área 1 de Madrid. Aten Primaria 2005; 36(2): 110-112.

33. Mingote Adán JC, Moreno-Jiménez B, Gálvez Herrer M. Desgaste profesional y salud de los profesionales médicos: revisión y propuestas de prevención. Med Clin (Barc) 2004; 123(7): 265-270.

34. Moreno-Jiménez B, Gálvez Herrer M, Garrosa Hernández E, Mingote Adan JC. Nuevos planteamientos en la evaluación del burnout. La evaluación específica del desgaste profesional médico. Aten Primaria 2006; 38 (10): 544-549.

35. Rodríguez F, Blanco MA, Issa S, Romero L, Gay P. Relación de la calidad de vida profesional y el burnout en médicos de atención primaria. Aten Primaria 2005; 36(8): 442-7. 
36. Fernández ML, Villagrasa JR, Gamo M, Vázquez J, Cruz E, Aguirre MV, et al. Estudio de la satisfacción laboral y sus determinantes en los trabajadores sanitarios de un área de Madrid. Rev Esp Salud Publica 1995: 69: 487-97.

37. Demerouti E, Bakker AB, Nachreiner F, Schaufeli WB. A model of burnout and life satisfaction among nurses. Journal of Advanced Nursing, 2000; 32: 454-464.

38. Atance Martínez JC. Aspectos epidemiológicos del síndrome de burnout en el personal sanitario. Rev Esp Salud Pública 1997; 71: 293-303.

39. Madrid-Salud. Estudio de clima laboral en el Instituto de Adicciones. Publicación Institucional On Line. Disponible en: htpp://wwwmunimadrid.es

40. Duxbury ML, Amstrong GD, Drew D. Burnout Correlates. Nurs Res 1984; 33: 97.

41. Price L, Spence SH. Burnout symptoms amongst drug and alcohol service employees: Gender differences in the interaction between work and home stressors. Anxiety, Stress and Doping 1994; 7: 67-84.

42. Arbinaga F, Vázquez MM. Síndrome de burnout y estrategias de afrontamiento en los profesionales de los centros ambulatorios de atención a drogodependientes. Revista Española de Drogodependencias 2002; 4: 591-602.

43. Sibbald S, Bojke C, Gravelle H. National survey of job satisfaction and retirement intentions among general practicioners in England. BMJ 2003; 326: 22.

44. Cabezas Peña C. La calidad de vida de los profesionales. Form Med Contin Aten Prim 2000; Supl 7: 53-68.

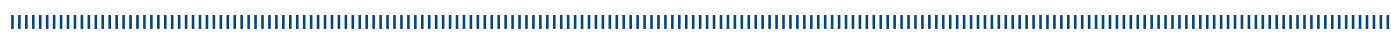

